\title{
Threshold behavior and electro-optical properties of twisted nematic layers with weak anchoring in the tilt and twist angle
}

\author{
R. Hirning, W. Funk, and H.-R. Trebin \\ Institut für Theoretische und Angewandte Physik der Universität Stuttgart, Pfaffenwaldring 57, \\ W-7000 Stuttgart 80, Federal Republic of Germany \\ M. Schmidt and H. Schmiedel \\ Sektion Physik der Universität Leipzig, Linnéstr. 5, 0-7010 Leipzig, Federal Republic of Germany
}

(Received 27 March 1991; accepted for publication 9 July 1991)

\begin{abstract}
Analytical and numerical calculations of threshold behavior and electro-optical characteristics in twisted chiral nematic layers are presented, when weak anchoring in the tilt and twist angle of the director is assumed. An analytical expression for the effective twist angle and the Fréedericksz threshold voltage is derived. In cells with bistabilities, we investigate the influence of the anchoring parameters and device parameters on the width of the hysteresis. Using the 4X4-matrix formalism of Berreman [J. Opt. Soc. Am. 62, 502 (1972)], we demonstrate the influence of the weak anchoring on the transmission-versus-voltage characteristic.
\end{abstract}

\section{INTRODUCTION}

The director configuration in twisted nematic layers like $\mathrm{TN},{ }^{1} \mathrm{OMI}^{2}$ or $\mathrm{SBE}^{3}$ cells is determined by the following three major features: First, the elastic forces in the liquid crystal, described by the well-known Frank-OseenZocher free-energy density ${ }^{4}$; second, the influence of an external applied voltage modeled through an electric energy term of the form $\frac{1}{2} \mathbf{D E}$, where $\mathbf{D}$ is the displacement vector and $\mathbf{E}$ the internal electric field vector; and third, the anchoring of the director at the substrate boundaries of the layer. Earlier work in analytical and numerical calculations used either strong ${ }^{5,6}$ or weak $^{7-9}$ anchoring in the tilt angle of the director (polar anchoring), i.e., its orientation with respect to the surface normal is either fixed or can vary with the applied voltage. Several authors report theoretical and experimental results ${ }^{10,11}$ on weak anchoring effects only in the twist angle (azimuthal anchoring), where the director is parallel to the surface but is allowed to leave its preferred orientation with respect to an axis in the surface (easy axis) under the influence of an applied voltage. This axis can be realized, for instance, by rubbing the surface carefully in one direction. Recently, some experimental studies of both types of anchoring have been published, ${ }^{12-14}$ showing that typical values of the anchoring energy are in the range $10^{-6}-10^{-5} \mathrm{~N} / \mathrm{m}$ for homeotropically anchored nematics and $10^{-6}-10^{-3} \mathrm{~N} / \mathrm{m}$ for planarly oriented nematics. In the last case, the azimuthal anchoring energy is one order of magnitude smaller than the polar energy. As far as we know, only the simulation program DIMOS $^{15}$ incorporates both types of anchoring.

In this paper the two kinds of anchoring are combined and studies are presented of the influence on the Fréedericksz threshold voltage, on the hysteresis width, and on the optical properties in such cells.

\section{THEORY}

We consider a nematic cell of thickness $d$ located between the planes $z=0$ and $z=d$ of a Cartesian coordinate system and mirror symmetric with respect to the middle plane $z=d / 2$. The director $\mathbf{n}$ is described by the tilt angle $\theta$ (measured from the layer normal) and the twist angle $\varphi$. The dielectric constants parallel and perpendicular to the director are denoted by $\varepsilon_{\|}$and $\varepsilon_{1}$, and we assume $\Delta \varepsilon$ $=\varepsilon_{\|}-\varepsilon_{1}>0$. The elastic constants for splay, twist, and bend are denoted by $k_{11}, k_{22}$, and $k_{33}$, respectively. The pitch of the material induced through a chiral dopant is named $p_{0}$.

$$
\begin{aligned}
& \text { Using the abbreviations } \\
& \begin{array}{l}
a_{1}=k_{33} \cos ^{2} \theta+k_{11} \sin ^{2} \theta, \\
a_{2}=\left(k_{33} \cos ^{2} \theta+k_{22} \sin ^{2} \theta\right) \sin ^{2} \theta, \\
a_{3}=\left(2 \pi / p_{0}\right) k_{22} \sin ^{2} \theta, \\
a_{4}=\varepsilon_{0}\left(\varepsilon_{1}+\Delta \varepsilon \cos ^{2} \theta\right)
\end{array}
\end{aligned}
$$

( $\varepsilon_{0}$ is the dielectric constant in vacuum), the free-energy density in the bulk can be written as ${ }^{4}$

$$
f_{B}=\frac{1}{2} a_{1} \theta^{\prime 2}+\frac{1}{2} a_{2} \varphi^{\prime 2}-a_{3} \varphi^{\prime}-\frac{1}{2} a_{4} \Phi^{\prime 2},
$$

where the prime indicates differentiation with respect to $z$ and where $\Phi$ is the electric potential inside the layer. Note that the last term represents the electric contribution $\frac{1}{2} \mathbf{D E}$, when we assume a dielectric material law for uniaxial nematics in the form

$$
D_{i}=\left(\varepsilon_{1} \delta_{i j}+\Delta \varepsilon n_{i} n_{j}\right) E_{j},
$$

with $E_{j}=-\partial_{j} \Phi$. Because we are interested in the director configuration for fixed voltage, we must add the electric contribution with the minus sign. ${ }^{16}$

The weak anchoring in the tilt and twist angle is described by a surface free-energy of the Rapini-Papoular type $^{17}$ :

$$
\begin{aligned}
& F_{S}(z=0)=\frac{1}{2} C_{\theta} \sin ^{2}\left(\theta-\theta_{p}\right)+\frac{1}{2} C_{\varphi} \sin ^{2} \varphi, \\
& F_{S}(z=d)=\frac{1}{2} C_{\theta} \sin ^{2}\left(\theta-\theta_{p}\right)+\frac{1}{2} C_{\varphi} \sin ^{2}\left(\varphi-\varphi_{p}\right)
\end{aligned}
$$


The factors $C_{\theta}$ and $C_{\varphi}$ measure the anchoring strength in the tilt and twist angle, respectively, $\theta_{p}$ (pretilt) describes the preferred tilt of the director at the surface, and $\varphi_{p}$ (pretwist) is the difference between the preferred orientation at the top and bottom surface in the twist angle. The influence of the surface is restricted to the place of the aligning substrate.

The total free energy per unit area of the cell is now given by

$$
F=\int_{0}^{d} f_{B} d z+F_{S}(0)+F_{S}(d) .
$$

\section{A. Fréedericksz threshold voltage (analytical)}

In this section we restrict ourselves to the case where the pretilt is equal to $90^{\circ}$. First, we investigate the director configuration if no electrical voltage is applied. The Eulerian equations resulting from the condition that the first variation of $F$ in Eq. (5) vanishes, split into the bulk equations and the surface torque balances. The bulk equations are given by

$$
\begin{gathered}
\left(k_{11} \sin ^{2} \theta+k_{33} \cos ^{2} \theta\right) \theta^{\prime \prime}-\left(k_{33}-k_{11}\right) \sin \theta \cos \theta \theta^{\prime 2} \\
-\left[k_{33}+2\left(k_{22}-k_{33}\right) \sin ^{2} \theta\right] \sin \theta \cos \theta \varphi^{\prime 2} \\
-\left(4 \pi / p_{0}\right) k_{22} \sin \theta \cos \theta \varphi^{\prime}=0
\end{gathered}
$$

and

$$
\begin{aligned}
& \left(k_{33} \cos ^{2} \theta+k_{22} \sin ^{2} \theta\right) \sin ^{2} \theta \varphi^{\prime}-\left(2 \pi / p_{0}\right) k_{22} \sin ^{2} \theta \\
& =\text { const. }
\end{aligned}
$$

The surface torque balances for tilt and twist, respectively, are given by

$$
\left.\frac{\partial f_{B}}{\partial \theta^{\prime}}\right|_{z=0}=C_{\theta} \sin \left(\theta-\theta_{p}\right) \cos \left(\theta-\theta_{p}\right)
$$

and

$$
\left.\frac{\partial f_{B}}{\partial \varphi^{\prime}}\right|_{z=0}=C_{\varphi} \sin \varphi \cos \varphi .
$$

Corresponding equations hold for $z=d$. Equations (6) and (7) are solved by the distribution $\theta(z)=90^{\circ}$, $\varphi^{\prime}(z)=$ const. Hence the director is planar and uniformly twisted with

$$
\varphi(z)=\varphi_{0}+(z / d) \varphi_{\mathrm{eff}},
$$

where the effective total twist angle is given by

$$
\varphi_{\text {eff }}=\varphi_{p}-2 \varphi_{0},
$$

and $\varphi_{0}=\varphi(0)$ is calculated from Eq. (9).

Let us discuss the influence of the weak coupling in the twist. We first note that the deviation angle from the pretwist direction, $\varphi_{0}$, vanishes if the pitch due to the chiral additive matches the pretwist given by the surface treatment, i.e., $\varphi_{p}=2 \pi d / p_{0}$. Therefore, the effective twist in this case is equal to the pretwist and weak twist coupling does not affect the director distribution for zero voltage. How- ever, if $\varphi_{p}>2 \pi d / p_{0}$, the effective twist becomes smaller than the pretwist, $\varphi_{\mathrm{eff}}<\varphi_{p}$, and vice versa. For strong twist anchoring we obtain $\varphi_{\text {eff }}=\varphi_{p}$.

To calculate the Fréedericksz threshold we modify the ansatz used for small deformations in the case of strong tilt and twist anchoring ${ }^{18}$ :

$$
\theta(z)=\theta_{m} \sin [\pi z /(d+2 b)],
$$

where $\theta_{m}$ denotes the midplane tilt angle and $b$ is a fictive extrapolation length to describe the weak tilt anchoring. Substituting Eqs. (12) and (10) into $f_{B}$ and integrating over the cell thickness yields the free energy as a function of $\theta_{m}$ and $\varphi_{0}$. The equations of the variational principle are then solved in the limit $\theta_{m} \rightarrow 90^{\circ}$, which leads to the Freedericksz threshold voltage $V_{F}$ at which the deformation starts. ${ }^{18}$ We obtain

$$
V_{F}=\sqrt{\frac{k_{11} \pi^{2} R^{2}+\varphi_{\mathrm{eff}}\left[\varphi_{\mathrm{eff}}\left(k_{33}-2 k_{22}\right)+4 k_{22} \pi d / p_{0}\right]}{\varepsilon_{0}\left(\varepsilon_{\|}-\varepsilon_{1}\right)}},
$$

where $\varphi_{\text {eff }}$ is determined by Eq. (11), $\varphi_{0}$ and $R$ are the solutions of the transcendental equations,

$$
\varphi_{p}-2 \varphi_{0}-\frac{2 \pi d}{p_{0}}=\frac{C_{\varphi} d}{k_{22}} \sin \varphi_{0} \cos \varphi_{0}
$$

and

$$
\cot \left(\frac{\pi R}{2}\right)=\pi R \frac{k_{11}}{C_{\theta} d} .
$$

Note that Eq. (13) involves weak anchoring for both the tilt and twist. For the case of strong twist anchoring we obtain the same result as Becker et al., ${ }^{6}$ who expanded the corresponding differential equations to calculate $V_{F}$.

Finally, we remark that the Freedericksz threshold voltage is affected by weak twist coupling only if the pitch does not match the pretwist. The weak twist anchoring can then increase or decrease the Fréedericksz threshold voltage depending on the ratios $\left(2 \pi d / p_{0}\right) / \varphi_{p}$ and $k_{33} / k_{22}$. In contrast to this behavior, a weak tilt coupling always decreases $V_{F}$ as was already mentioned by Becker et al. ${ }^{6}$

\section{B. Numerical procedure}

The first step in the calculation of optical properties consists of the determination of the director configuration. To this end, we transform the Euler-Lagrange equations resulting from the extremalization of the bulk free energy into a system of Hamilton equations by performing a Legendre transformation with respect to the variables $\theta, \varphi$, and $\Phi$ :

$$
\begin{aligned}
& \theta^{\prime}=\frac{\partial f_{B}^{L}}{\partial T}=\frac{T}{a_{1}}, \\
& \varphi^{\prime}=\frac{\partial f_{B}^{L}}{\partial P}=\frac{P+a_{3}}{a_{2}}, \\
& \Phi^{\prime}=\frac{\partial f_{B}^{L}}{\partial U}=-\frac{U}{a_{4}},
\end{aligned}
$$




$$
\begin{gathered}
T^{\prime}=-\frac{\partial f_{B}^{L}}{\partial \theta}=\frac{b_{1}}{2 a_{1}^{2}} T^{2}-\frac{b_{3}}{a_{2}}\left(P+a_{3}\right) \\
+\frac{b_{2}}{2 a_{2}^{2}}\left(P+a_{3}\right)^{2}-\frac{b_{4}}{2 a_{4}^{2}} U^{2}, \\
P^{\prime}=-\frac{\partial f_{B}^{L}}{\partial \varphi}=0, \\
U^{\prime}=-\frac{\partial f_{B}^{L}}{\partial \Phi}=0,
\end{gathered}
$$

where $f_{B}^{L}$ is the Legendre transform of $f_{B}$ :

$$
f_{B}^{L}(\theta, T, P, U)=\theta^{\prime} T+\varphi^{\prime} P+\Phi^{\prime} U-f_{B}
$$

and $b_{i}=\partial a_{i} / \partial \theta, i=1,4$, and $T, P, U$ are the conjugated momenta:

$$
\begin{aligned}
& T=\frac{\partial f_{B}}{\partial \theta^{\prime}}=a_{1} \theta^{\prime}, \\
& P=\frac{\partial f_{B}}{\partial \varphi^{\prime}}=a_{2} \varphi^{\prime}-a_{3}, \\
& U=\frac{\partial f_{B}}{\partial \Phi^{\prime}}=-a_{4} \Phi^{\prime} .
\end{aligned}
$$

As $\varphi$ and $\Phi$ are cyclic variables, the corresponding momenta $P$ and $U$ represent integration constants for the problem, where $U$ is equal to the $z$ component of the displacement vector $\mathbf{D}$. To these equations we have to add the boundary conditions (8) and (9), expressed in the new variables at $z=0$ :

$$
\begin{aligned}
& T=+\frac{\partial F_{S}}{\partial \theta}=C_{\theta} \sin \left(\theta-\theta_{p}\right) \cos \left(\theta-\theta_{p}\right), \\
& P=+\frac{\partial F_{S}}{\partial \varphi}=C_{\varphi} \sin \varphi \cos \varphi,
\end{aligned}
$$

and at $z=d$ :

$$
\begin{aligned}
& T=-\frac{\partial F_{S}}{\partial \theta}=-C_{\theta} \sin \left(\theta-\theta_{p}\right) \cos \left(\theta-\theta_{p}\right), \\
& P=-\frac{\partial F_{S}}{\partial \varphi}=-C_{\varphi} \sin \left(\varphi-\varphi_{p}\right) \cos \left(\varphi-\varphi_{p}\right) .
\end{aligned}
$$

Equations (16), (19), and (20) represent a nonlinear boundary-value problem, which we solve numerically by a multidimensional shooting method using standard library routines.

The second step is to solve the Maxwell equations inside the layer. For this problem we use the $4 \times 4$ formalism of Berreman. ${ }^{19-21}$ To decide whether our numerical calculated optical transfer matrix of the nematic layer is correct, we use the condition ${ }^{22}$ that the modulus of the determinant for this matrix must be equal to 1 , which expresses the law of conservation of energy.

Our main emphasis was to implement an efficient code which works properly and fast over a broad range of material and device parameters. On an IBM PC/AT with 10 $\mathrm{MHz}$, for instance, the calculation of an electro-optical

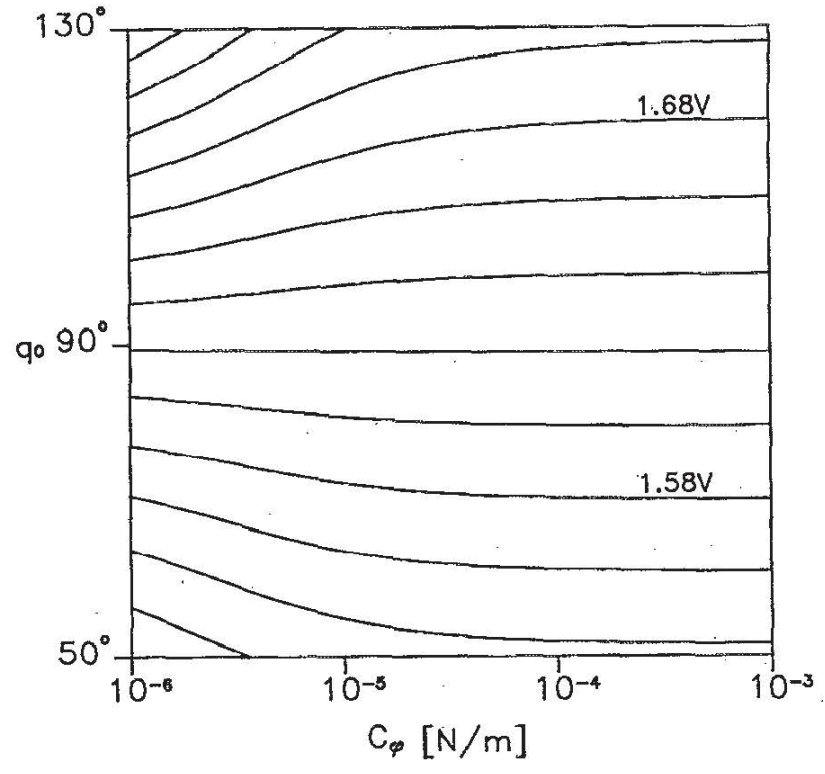

FIG. 1. Fréedericksz threshold voltage for a $90^{\circ}$ TN cell as a function of $C_{\varphi}$ and $q_{0}$. The difference between neighboring lines is $0.02 \mathrm{~V}$. Material parameters are $k_{33} / k_{11}=0.8, k_{22} / k_{11}=0.45, \varepsilon_{\|} / \varepsilon_{1}=2.3$; the cell thickness is $5.6 \mu \mathrm{m}$.

characteristic needs about $10 \mathrm{~min}$ independent of the existence of bistabilities.

\section{NUMERICAL RESULTS AND DISCUSSION}

\section{A. Freedericksz threshold voltage (numerical)}

To test the correctness of the analytical expression for the Freedericksz threshold voltage, we have calculated it for a $90^{\circ} \mathrm{TN}$ cell with $q_{0}=2 \pi d / p_{0}$ and $C_{\varphi}$ as variables, $\theta_{p}=90^{\circ}$, and with fixed values of $C_{\theta}=10^{-4} \mathrm{~N} / \mathrm{m}$. Figure 1 shows the lines of constant threshold voltage resulting from our numerical calculations. (Note that we measure

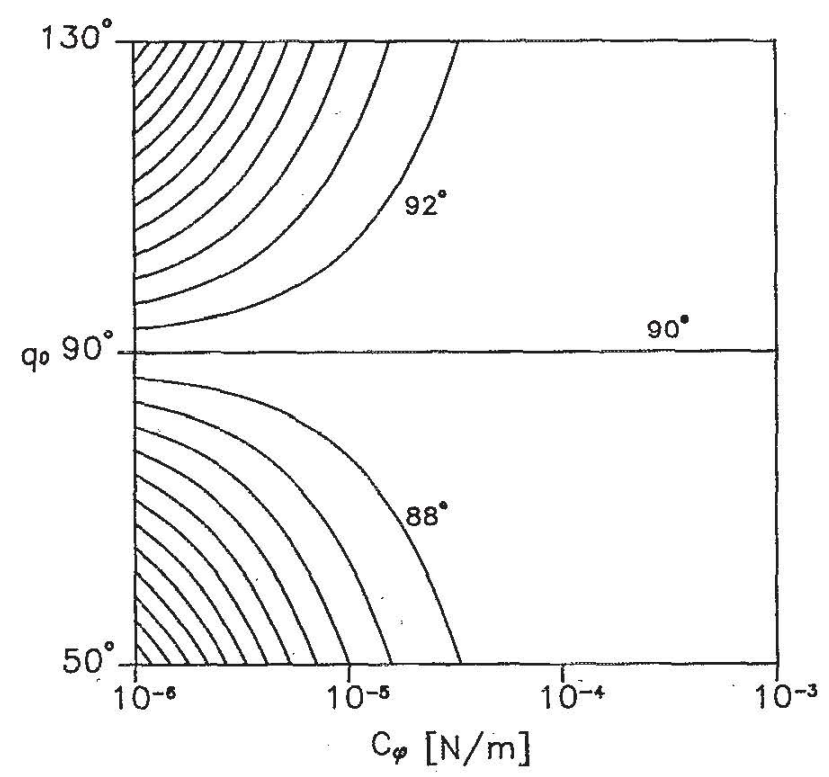

FIG. 2. Effective twist angle for a $90^{\circ} \mathrm{TN}$ cell as a function of $C_{\varphi}$ and $q_{0}$. The difference between neighboring curves is $2^{\circ}$. Material parameters are as in Fig. 1. 


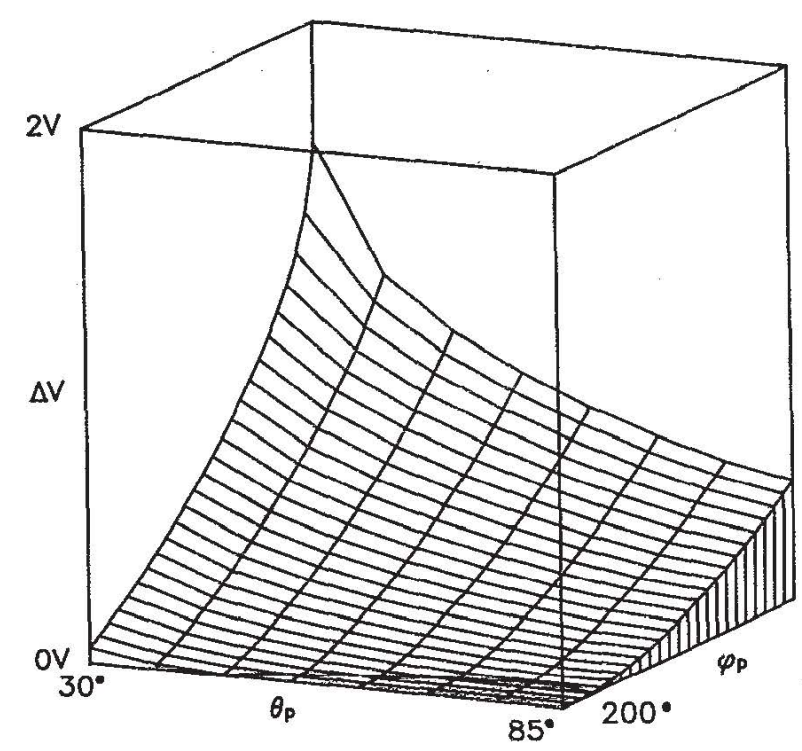

FIG. 3. Hysteresis width for a $240^{\circ}$ STN cell as a function of $\varphi_{p}$ and $\theta_{p}$; the anchoring strength $C_{\varphi}$ is $10^{-4} \mathrm{~N} / \mathrm{m}$. Material parameters are $k_{33} / k_{11}=2.1, k_{22} / k_{11}=0.4, \varepsilon_{\|} / \varepsilon_{1}=2.0$; the cell thickness is $5.6 \mu \mathrm{m}$.

$q_{0}$ in rad, e.g., $q_{0}=90^{\circ}$ means that the intrinsic pitch is identical with the extrinsic pitch imposed by the boundary conditions.) The values of $V_{F}$ resulting from the analytical calculations [see Eq. (13)] are identical with the numerical ones and show the behavior predicted in Sec. II A.

\section{B. Effective twist angle}

In Fig. 2 we give the curves of constant effective twist angle $\varphi_{\text {eff }}$ at zero applied voltage $V$ as a function of $q_{0}$ and $C_{\varphi}$ resulting from our numerical calculations. It is seen that $\varphi_{\mathrm{eff}}$ increases with decreasing $C_{\varphi}$ if $q_{0}$ is greater than the pretwist $\varphi_{p}$; on the other hand, if $q_{0}$ is smaller than the $\varphi_{p}, \varphi_{\mathrm{eff}}$ is decreasing with decreasing $C_{\varphi}$. Analogously to

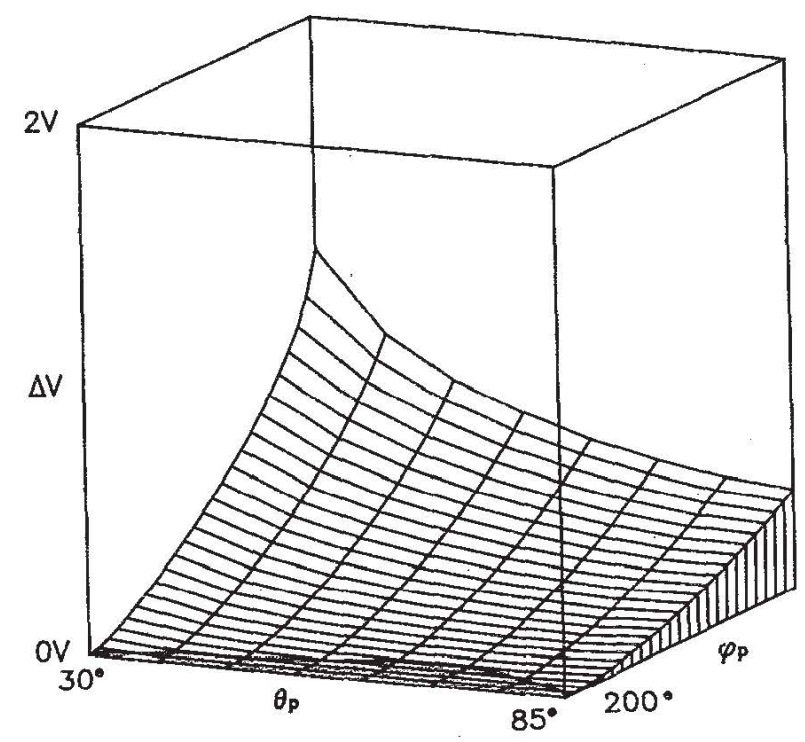

$320^{\circ}$

FIG. 4. Same plot as in Fig. 3 with $C_{\varphi}=10^{-5} \mathrm{~N} / \mathrm{m}$.

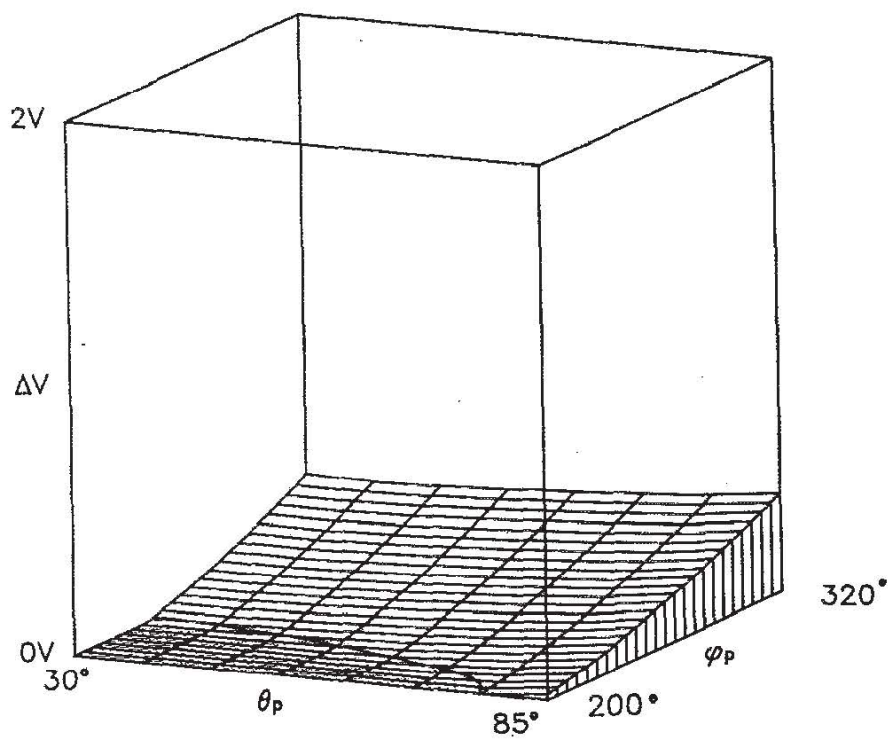

FIG. 5. Same plot as in Fig. 3 with $C_{\varphi}=10^{-6} \mathrm{~N} / \mathrm{m}$.

the preceding section the analytical values [resulting from Eqs. (11) and (14)] agree with the numerical ones.

\section{Width of the hysteresis}

In highly twisted cells with nonzero pretilt, there is the possibility of bistable director configurations, ${ }^{8,18,23-27}$ which becomes evident by a hysteresis in the $\theta_{m}$-versus-voltage curve. In Figs. 3-5, we have plotted the width of the hysteresis $\Delta V$ as a function of $\theta_{p}$ and $\varphi_{p}$ for $C_{\varphi}=10^{-4}, 10^{-5}$, and $10^{-6} \mathrm{~N} / \mathrm{m} . C_{\theta}$ was held constant at $10^{-4} \mathrm{~N} / \mathrm{m}$, and we have chosen $q_{0}$ equal to $\varphi_{p}$. We get a

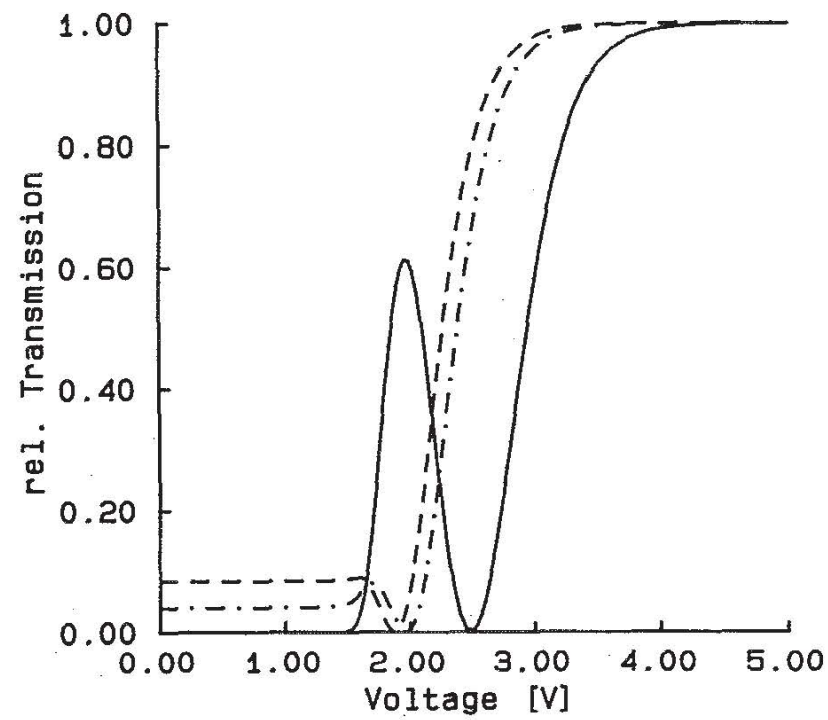

FIG. 6. Transmission-vs-voltage characteristic for a $90^{\circ}$ TN cell of thickness $d=5.6 \mu \mathrm{m}$; the material parameters are the same as in Fig. 1; the optical birefringence indices are $n_{0}=1.5$ and $n_{e}=1.65$, the wavelength $\lambda$ is $550 \mathrm{~nm}$, polarizer and analyzer are parallel to the preferred direction in the twist angle at $z=0$; the solid line represents the curve for $C_{\varphi}$ $=10^{-6} \mathrm{~N} / \mathrm{m}$, the dashed-dotted line for $C_{\varphi}=10^{-5} \mathrm{~N} / \mathrm{m}$, and the dashed line for $C_{\varphi}=10^{-4} \mathrm{~N} / \mathrm{m} ; C_{\theta}$ is $10^{-4} \mathrm{~N} / \mathrm{m}$ in each case. 


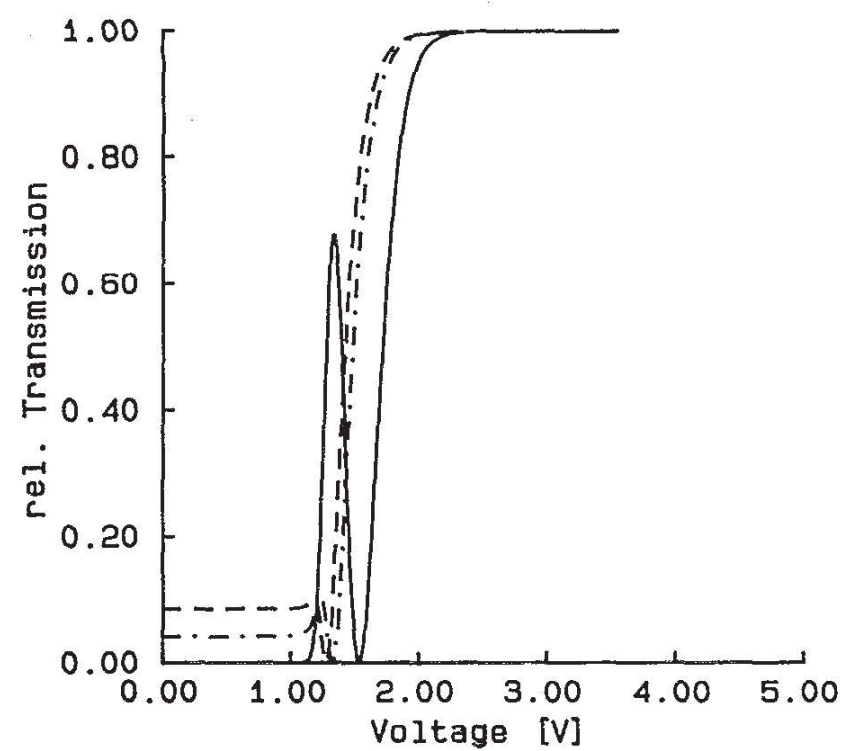

FIG. 7. Same curves as in Fig. 6 with $C_{\theta}=10^{-5} \mathrm{~N} / \mathrm{m}$.

monotonic increase of $\Delta V$ in the variable $\varphi_{p}$. Further, it can be seen that for fixed $\varphi_{p}$ and $\theta_{p}$ the width of the hysteresis falls with decreasing $C_{\varphi}$. If $C_{\varphi}$ gets smaller the onset of the hysteresis increases for $\varphi_{p}$ and decreases for $\theta_{p}$.

\section{Transmission-versus-voltage curves}

One of the most important features for a twisted nematic layer is its transmission-versus-voltage characteristic. In Figs. 6 and 7 we show these curves for a TN cell with $C_{\theta}=10^{-4} \mathrm{~N} / \mathrm{m}$ and $C_{\theta}=10^{-5} \mathrm{~N} / \mathrm{m}$. The parameter of the curves is $C_{\varphi}$, the wave vector of the incident light with

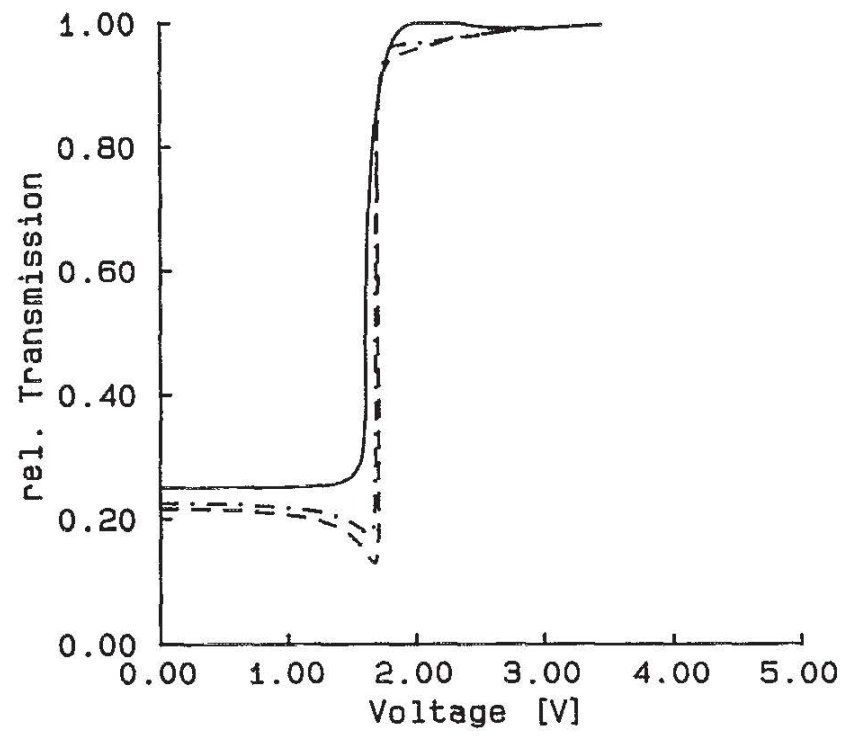

FIG. 8. Transmission-vs-voltage characteristic for a $240^{\circ}$ SBE cell of thickness $d=5.6 \mu \mathrm{m}$; the material parameters are the same as in Fig. 3; the optical birefringence indices are $n_{o}=1.49$ and $n_{e}=1.63$, the wavelength $\lambda$ is $589 \mathrm{~nm}$, polarizer and analyzer are oriented parallel exactly in the middle between the preferred directions in the twist angle at $z=0$ and $z=d$; the solid line represents the curve for $C_{\varphi}=10^{-6} \mathrm{~N} / \mathrm{m}$, the dasheddotted line for $C_{\varphi}=10^{-5} \mathrm{~N} / \mathrm{m}$, and the dashed line for $C_{\varphi}=10^{-4} \mathrm{~N} / \mathrm{m}$; $C_{\theta}$ is $10^{-4} \mathrm{~N} / \mathrm{m}$ in each case.

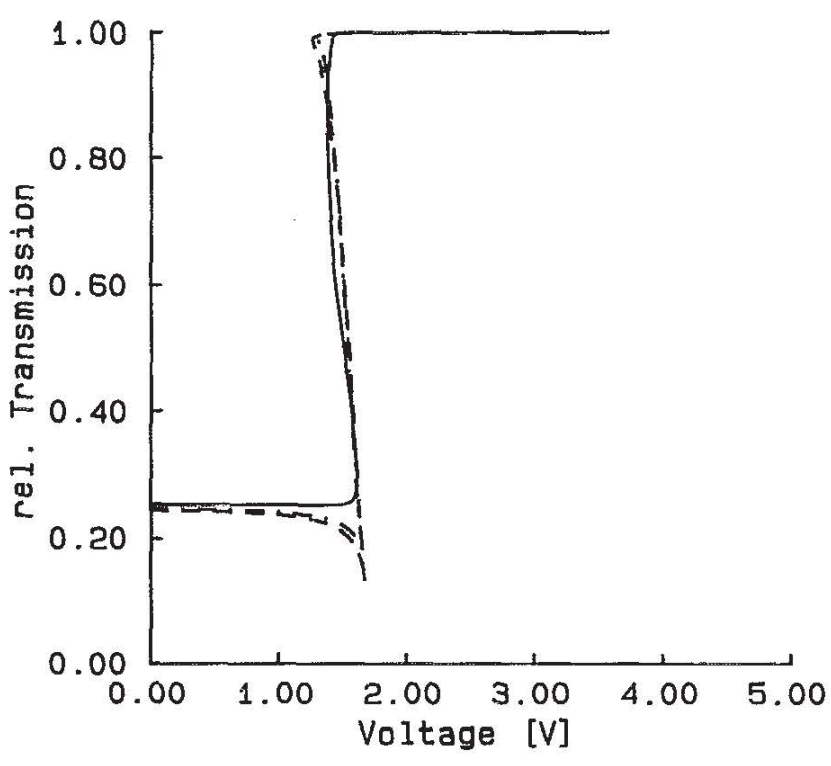

FIG. 9. Same curves as in Fig. 8 with $C_{\dot{\theta}}=10^{-5} \mathrm{~N} / \mathrm{m}$.

$\lambda=550 \mathrm{~nm}$ is normal to the cell surface, and we assume a pretilt $\theta_{p}$ of $89^{\circ}$. A characteristic property in both figures is the peak below the optical threshold voltage, which is defined as that voltage for which the Mauguin parameter $M$ in the middle of the cell becomes greater than unity. ${ }^{28}$ The peak appears at the voltage for which the voltage-depen dent optical path difference $\overline{\Delta n} d$ averaged over the whole cell causes a constructive interference of the extraordinary and ordinary optical normal modes. This proves that the peak is a birefringence effect. Our calculations show further that the height of the peak is raised by reducing $C_{\varphi}$, whereas the width is getting broader for increasing $C_{\theta}$. The wavelength $\lambda$ of the incident light influences the peak, too; with increasing $\lambda$ the height increases slightly, the location is slightly shifted to smaller voltages, whereas the shape remains almost unchanged.

Figures 8 and 9 show the same curves as in Figs. 6 and 7 but now for a cell with a pretilt of $70^{\circ}$, a pretwist $\varphi_{p}$ of $240^{\circ}$, and light of $\lambda=589 \mathrm{~nm}$. We have chosen the optical path difference in such a way that the cell works in the SBE mode. It is seen that a weak anchoring in the twist reduces the hysteresis (see the preceding section), whereas a weak anchoring in the tilt enhances it.

${ }^{1}$ M. Schadt and W. Helfrich, Appl. Phys. Lett. 18, 127 (1971).

${ }^{2}$ M. Schadt and F. Leenhouts, Appl. Phys. Lett. 50, 236 (1987).

${ }^{3}$ T. J. Scheffer and J. Nehring, J. Appl. Phys. 58, 3022 (1985).

${ }^{4}$ P. G. de Gennes, The Physics of Liquid Crystals (Clarendon, Oxford, 1974).

${ }^{5}$ G. Baur, F. Windscheid, and D. Berreman, Appl. Phys. 8, 101 (1975).

${ }^{6}$ M. E. Becker, J. Nehring, and T. J. Scheffer, J. Appl. Phys. 57, 4539 (1985).

${ }^{7}$ J. Nehring, A. Kmetz, and T. J. Scheffer, J. Appl. Phys. 47, 850 (1976). ${ }^{8}$ H. A. van Sprang and P. A. Breddels, J. Appl. Phys. 60, 968 (1986).

${ }^{9}$ G. Haas, H. Wöhler, M. Fritsch, and D. Mlyinski, 17. Freiburger Arbeitstagung Flüssigkristalle, 1987.

${ }^{10} \mathrm{G}$. Barbero, E. Miraldi, C. Oldano, M. L. Rastello, and P. Taverna Valabrega, J. Phys. (Paris) 47, 1411 (1986).

${ }^{11}$ S. Faetti, M. Gatti, V. Palleschi, and T. J. Sluckin, Phys. Rev. Lett. 55, 1681 (1985).

${ }^{12}$ T. Sugiyama, S. Kuniyasu, and D. Seo, Jpn. J. Appl. Phys. 29, 2045 (1990). 
${ }^{13}$ K. Ogawa, N. Mino, and K. Nakajima, Jpn. J. Appl. Phys. 29, L1689 (1990).

${ }^{14}$ M. Blinov, A. Kabayenkov, and A. Sonin, Liq. Cryst. 5, 645 (1989). ${ }^{15} \mathrm{M}$. Becker, Electronic Displays, Wiesbaden, 1988.

${ }^{16}$ R. Thurston and D. Berreman, J. Appl. Phys. 52, 508 (1981).

${ }^{17}$ A. Rapini and M. Papoular, J. Phys. (Paris) Colloq. 30, C4-54 (1969).

${ }^{18}$ M. Schmidt and H. Schmiedel, Mol. Cryst. Liq. Cryst. 167, 89 (1989).

${ }^{19}$ D. W. Berreman, J. Opt. Soc. Am. 62, 502 (1972).

${ }^{20} \mathrm{~K}$. Eidner, G. Mayer, M. Schmidt, and H. Schmiedel, Mol. Cryst. Liq. Cryst. 172, 191 (1989).
${ }^{21}$ H. Wöhler, G. Haas, M. Fritsch, and D. Mlynski, J. Opt. Soc. Am. A 5, 1554 (1988).

${ }^{22}$ M. Schmidt and H. Schmiedel, Mol. Cryst. Liq. Cryst. 172, 223 (1989).

${ }^{23}$ S. Saito, M. Kamihara, and S. Kobayashi, Mol. Cryst. Liq. Cryst. 139, 171 (1986).

${ }^{24}$ T. J. Scheffer and J. Nehring, Appl. Phys. Lett. 45, 1021 (1984).

${ }^{25}$ D. W. Berreman and W. R. Heffner, J. Appl. Phys. 52, 3032 (1981).

${ }^{26}$ H. K. Singh, S. C. Jain, and S. Chandra, Liq. Cryst. 5, 1373 (1989).

${ }^{27}$ E. P. Raynes, Mol. Cryst. Liq. Cryst. Lett. 4, 1 (1986).

${ }^{28}$ C. Van Doorn and J. Heldens, Phys. Lett. 47A, 135 (1974). 